\title{
Eco-Efficiency Measurement and Material Balance Principle: an Application in Power Plants Malmquist Luenberger Index
}

\author{
Behrouz Arabia $^{a}$,Susila Munisamy Doraisamy ${ }^{\mathrm{b}}$, Ali Emrouznejad ${ }^{\mathrm{c}}$, Alireza Khoshroo ${ }^{\mathrm{d}}$ \\ ${ }^{\text {a }}$ Faculty of Economics and Administration, University of Malaya, Malaysia \\ ${ }^{\mathrm{b}}$ Faculty of Economics and Administration, University of Malaya, Malaysia \\ ${ }^{c}$ Aston Business School, Aston University, Birmingham, UK \\ ${ }^{\mathrm{d}}$ Department of Agricultural Engineering, Faculty of Agriculture, University of Yasouj, Yasouj, Iran
}

\begin{abstract}
Incorporating Material Balance Principle in industrial and agricultural performance measurement systems with pollutant factors has been on the rise in recent years. Many conventional methods of performance measurement have proven incompatible with the material flow conditions. This study will address the issue of eco-efficiency measurement adjusted for pollution, taking into account materials flow conditions and the Material Balance Principle (MBP) requirements, in order to provide 'real' measures of performance that can serve as guides when making policies. We develop a new approach by integrating slacks-based measure to enhance the Malmquist Luenberger Index by a material balance condition that reflects the conservation of matter. This model is compared with a similar model, which incorporates MBP using the trade-off approach to measure productivity and eco-efficiency trends of power plants. Results reveal similar findings for both models substantiating robustness and applicability of the proposed model in this paper.
\end{abstract}

Keywords: Data Envelopment Analysis; Material Balance Principle; Slacks-based Model; ecoefficiency; Malmquist Luenberger Index

\section{Introduction}

Over the past three decades, parallel to predominant international concerns about environmental disasters caused by overexploiting natural resources and polluting the nature, researchers have

\footnotetext{
${ }^{1}$ Corresponding Author: Ali Emrouznejad, Aston Business School, Aston University, Birmingham, UK, a.emrouznejad@aston.ac.uk
} 
focused on performance measurement systems with pollutant factors, taking into account material flow conditions [1]. The first law of thermodynamics, i.e. the law of conservation of matter and energy, emphasizes that in any production system "what goes in must come out" [2], which is known as the Material Balance Principle (MBP). Thus, in order for performance measurement methods to be more precise, the production function used should be compatible with MBP.

MBP cemented its status in performance measurement studies when bad outputs or pollutants became an unavoidable factor in efficiency measurement systems addressing environmental concerns [3]. There are two popular approaches toward frontier-based efficiency measurement models with bad outputs distinguished by Tyteca [4]; i.e., parametric and non-parametric ones. Parametric frontier methods are known as Stochastic Frontier Approach (SFA), and the nonparametric group is well known as Data Envelopment Analysis (DEA). Nevertheless, Lauwers [1] expressed that MBP has been neglected in the majority of previous performance measurement studies and the non-parametric frontier models are no exception. As such, there is a potential to enhance eco-efficiency measurement methods and make them MBP-enabled. In this paper, we shall confine ourselves to non-parametric DEA models.

Coelli, Lauwers [3] showed that a number of previous DEA models such as hyperbolic efficiency measure in Fare, Grosskopf [5], input orientated efficiency measure in Färe, Grosskopf [6], and the three alternative efficiency measures used in Reinhard, Knox Lovell [7] are not compatible with MBP requirements (although under certain circumstances, a number of these models may happen to be compatible with MBP - See Coelli, Lauwers [3]). These types of models have used the distance function concept to measure eco-efficiency. Consequently, methods such as Malmquist Luenberger Index (MLI) measurement, which apply similar models to evaluate the eco-efficiency trend, may suffer from the same problem.

Chung, Färe [8] introduced MLI to portray eco-efficiency trend using Directional Distance Functions (DDF) to measure contemporary inefficiencies. DDF was originally introduced by Shephard, Gale [9] and has been adopted and applied since then by many other researchers in different ways [10-14]. Since MLI and DDF have been recognized as non-parametric frontier methods for eco-efficiency measurement incorporating pollutant as bad outputs, the material flow conditions and MBP are generally expected to be applicable to these types of methods. 
However, there are major inadequacies in the traditional methods of eco-efficiency measurement. This study will address the issue of eco-efficiency measurement, taking into account materials flow conditions and the MBP requirements, in order to provide 'real' measures of performance that can serve as guides when making policies.

Therefore, in this paper, firstly we show that the popular DDF model, in its conventional form, is not MBP compatible. Next, we categorise different typical frontier models that can be found in production situations and introduce the conditions that should be imposed on the models to make them MBP-enabled. Further, we introduce a more comprehensive and flexible slacks-based model, which is consistent with MBP requirements. We enhance the Malmquist Luenberger Index by a material balance condition by making use of this slacks-based model. This new model is called the MBP Malmquist Luenberger Index. Finally, we run this new model on Iranian

power plants over an eight-year period to observe the eco-efficiency trend and discuss the applicability of the model.

The rest of the paper is organized as follows. The literature related to MBP and DEA ecoefficiency measurement methods in addition to previous efforts on incorporation of MBP requirements in DEA models are reviewed in Section 2. In Section 2.4, DDF and slacks-based models, adopted to incorporate bad outputs, are tested for MBP compatibility, and the conditions to be met to make them MBP-enabled are presented afterward. A more comprehensive slacksbased model, which is MBP enabled is introduced in Section 2.5. This new model is implemented in Section 3 to measure ML index for Iranian power plants. We also describe a DEA model that is adapted to incorporate MBP requirement by adding the trade-offs into a slacks-based model. Finally, results are presented and discussed in Section 4. Section 5 concludes the paper and indicates directions for future research.

\section{Methods}

\subsection{Material Balance Principle}

According to the first law of thermodynamics, matter can neither be created nor destroyed; this is known as the Material Balance Principle (MBP). For the first time, in 1969, Ayres and Kneese [15] added this concept to the glossary of economics. However, due to complicated operational 
problems involved in the research at that time, the combination of factors and processes, which allowed for curbing a high level of emissions only by a small increase in cost, could not be characterized clearly. However, the operational approach is far less complicated today.

An ecological system includes economic and social systems, which comprise production and consumption [16] or a natural environment determined by materials and energy flows including extraction, use, recycling and waste disposal [17]. The law of conservation of matter/energy is an essential biophysical condition expressing that flows from and into the environment are equivalent or balanced $[17,18]$. Hence, the fundamental concept of material balance condition essentially states that: "what goes in must come out". Linear programming models [19] described standardised, aggregated performance indicators for firms. Such indicators are adjusted for pollution and used in the frontier eco-efficiency models. Two different frontierbased eco-efficiency models were presented. First, the usual parametric (e.g. Stochastic Frontier Analysis) and nonparametric (e.g. Data Envelopment Analysis) frontier efficiency models were adjusted for pollution. The next model was called 'the Normalised Undesirable Output Approach', in which the relations between the ecological outcomes with respect to the economic outcomes were explained regardless of conventional inputs and desirable outputs. Lauwers [1] called these two types of models the 'Environmentally Adjusted Production Efficiency (EAPE) models and the 'Frontier Eco-Efficiency' (FEE) models. Based on some earlier empirical work by Lauwers, Van Huylenbroeck [20] on the nutrient balance in pig production, Coelli, Lauwers [3] worked out the theoretical and methodological aspects of the MBP incorporation.

Besides the usual technical efficiency and economic efficiency scores, Lauwers, Van Huylenbroeck [20] drew an analogy with the cost-minimizing models to calculate environmental efficiency scores from which allocative components could be derived. The material flow information was used in the same way as the price information was.

Like before, because of emerging concerns about the detrimental effects of humankind activities, a new approach toward incorporating undesirable outputs of production processes into the performance measurement methods came into focus for research. Whilst any performance measurement models should be compatible with the production technology and environmental outcomes, it is equally important for these models to be consistent with the material flow in the real system as well [1]. However, according to Lauwers [1], MBP has been neglected in the 
majority of previous studies and there is a need to fill this gap and thereby enhance the accuracy of the eco-efficiency measurement models. Lauwers, in 2009, proposed a frontier eco-efficiency modelling via incorporation of the Material Balance Principle (MBP) in a way that the environmental outcome derived from the production process would be similar to the economic outcome. The diagnostic power of eco-efficiency measurement is improved later by comparing the economic and environmental outcomes of the same technology.

Material Balance Principle is considered a linear relationship between inputs consumed and outcomes produced. Since mass cannot be destroyed in the production process, summation of the input nutrients, for example energy or emission that can be generated, should be equal to summation of the outcomes, including both good ones and bad ones. Murty, Robert Russell [21] opined that linearity is not a necessity: accordingly, they introduced some non-linear modeling for pollution generating technologies. Although there are some criticisms against the linear relationship, it sheds some light on the trade-offs between economic and environmental characteristics of conventional models. Nevertheless, these criticisms do not pose a challenge to the linearity of MBP formulation since it does reduce the complexity of the non-linear relationship between economic and environmental aspects of the system and makes it possible to the models and apply them [1].

In addition to non-linear pollution generating modeling, Murty, Robert Russell [21] and Pethig [22] modeled the abatement technologies incorporating material balance conditions. Furthermore, Färe, Grosskopf [23], applying a network approach, successfully formulated the abatement technology used in coal-fired power plants taking MBP conditions into consideration. Moreover, Coelli, Lauwers [3] formulated the abatement technologies in an MBP-enabled DEA approach.

After a comprehensive literature review on the evolution of MBP in his paper, Lauwers [1] discussed the diagnostic power and allocative aspects of MPB, which are ignored in the conventional eco-efficiency measurement methods, too. Coelli, Lauwers [3] have also introduced a workable method to analyze the economic-environmental trade-offs of a pollution generating system. In line with this, using the approach adopted by Coelli, Lauwers [3] to include input and output emission coefficients, Lauwers [1] maintains that social costs of pollution generating firms can be evaluated and minimized. The MBP-adjusted method, compared with the eco- 
efficiency frontier model, takes advantage of considering the underlying production technology and simultaneously explains its economic and ecological outcomes in an unbiased and clear manner. Hence, the gap between conventional concepts of production efficiency and ecoefficiency is bridged by using the MBP-adjusted method [1].

Recently, some other researchers employed incorporated MBP conditions in addition to DEA models to measure the efficiency of energy industries [23-26]. However, in this paper we discus general conditions for including MBP conditions into DEA, specially slack-back models and address the advantages of this series of models.

In summary, it is necessary for every eco-efficiency measurement tool to be compatible with MBP requirements; otherwise, a fundamental question will still remain to be answered in any study: is the production technology employed compatible with the nature of the industry? In this study, our focus is on non-parametric frontier DEA methods. We consider merits and flaws of the conventional methods and introduce an MBP-enabled DEA model. Next, a review of the related DEA literature is given in the following section.

\subsection{Eco-Efficiency Measurement DEA}

Farrell [27] initiated DEA by formulating the non-parametric technical efficiency. Later, Charnes, Cooper [28] introduced the first DEA linear programing model to measure the efficiency of constant return to scale technologies. Banker, Charnes [29], by introducing the first variable return to scale model, showed DEA has a capability to match different technologies. DEA has been applied in different areas related to Energy (example: Transportation [30], Agriculture [31], power plants [32], iron and steel sector [33]). Afterwards, many DEA models were introduced to the literature to handle different conditions in the efficiency measurement [34-36].

As a major tool for efficiency measurement, DEA was chosen for eco-efficiency measurement purposes, too. By conceiving the concept of bad outputs, a number of studies suggested approaches incorporating bad outputs in the efficiency measurement models [37-45]. It is worth noting that there are different types of bad outputs, which can be incorporated in efficiency measurement models; however, if the bad output is a pollutant, the measurement of efficiency is known as eco-efficiency. One of the most popular approaches toward incorporating the bad 
outputs in efficiency measurement - as addressed before - is the DDF model, introduced by Chung, Färe [8]. However, in spite of its popularity, DDF and many other eco-efficiency measurement models have been criticized seriously by Coelli, Lauwers [3] from the MBP point of view. The criticism culminated in their introducing an alternative DEA model, which was compatible with MBP. In the next section, we present previous MBP-enabled DEA models and discuss the conditions to be met for DEA models to become compatible with MBP.

\subsection{Material Balance Conditions and DEA Models}

To operationalize MBP, the MBP requirements are formulated as below:

Let $x \in \mathrm{P}^{I}, y \in \mathrm{P}^{J}$, and $z \in \mathrm{P}^{K}$ are inputs, outputs, and pollutant of a production unit. If production possibility set (PPS) is defined as: $P(x)=\{(y, z): x$ can produce $(y, z)\}$ and $a$ and $b$ are $(I \times l)$ and $(J \times l)$ non-negative coefficients of $x$ and $y$ respectively, which reflect the nutrient of the pollutant inside inputs and outputs based on MBP, the amount of pollutant should be written as:

$$
z=a^{\prime} x-b^{\prime} y
$$

This equation plays a key role in deciding if a model is compatible with MBP. Coelli, Lauwers [3] introduced an input-oriented constant return to scale model, which is compatible with MBP. This DEA-MBP model was successfully applied to measure environmental efficiency of pig finishing farms and later applied to electricity generation plants by Welch and Barnum [46]. This model was not the only successful approach toward incorporating MBP in DEA models. Färe, Grosskopf [23], by employing a network DDF model, which was consistent with MBP, measured the eco-efficiency of US coal-fired power plants using abatement technologies. In this paper, it is admitted that the weak disposability axiom ${ }^{2}$, as one of the core concepts of DDF, is hardly consistent with MBP. As a result, a facile condition was set so that utilization of abatement technologies ${ }^{3}$ would become likely.

Coelli, Lauwers [3] have already proven that some previously introduced models - in their general form- are not consistent with MBP. Here, we consider one of the most popular forms of

\footnotetext{
2 Weak disposability can be written as: $(y, z) \in P(x)$ and $0 \leq \theta \leq 1$ imply $(\theta y, \theta z) \in P(x)$, while free or strong disposability can be defined as: $(y, z) \in P(x)$ and $y^{\prime} \leq y$ imply $\left(y^{\prime}, z\right) \in P(x)$.

${ }^{3}$ In this case, whereas electricity is the good output and $\mathrm{So}_{2}$ is the bad one $b$ which is the nutrient coefficient of the good output is 0 . Sulfur is not a part of electricity.
} 
DDF model introduced by Chung, Färe [8] to demonstrate the incompatibility in question. As other directional distance models, the DDF model seeks the largest amount of $\theta$, which can keep the vector $(x, y+\theta y, z-\theta z)$ inside the PPS. If we apply this vector to equation (1), we will have: $z-$ $\theta z=a^{\prime} x-b^{\prime}(y+\theta y)$. Then, with some simplifications, we obtain: $z=a^{\prime} x-b^{\prime} y-\theta\left(b^{\prime} y-z\right)$, here MBP holds only if $\theta\left(b^{\prime} y-z\right)=0$. If $\theta=0$, then the unit has been located on the frontier; thus the MBP holds. However, for non-efficient units in the interior of the PPS, the MBP conditions are not valid. On the other hand, if $b^{\prime} y-z=0$, then $b^{\prime} y=z$. It implies that the actual pollutant amount should be equal to the pollutant released by the good outputs. This condition occurs only in very limited circumstances because in the production technologies it is very hard to find an analogue of equal amount of generated pollutant and the pollutant that is generated by the good outputs. This situation is worsen when $b=0$, for example when electricity is the sole output. If so, $b^{\prime} y=z$ implies $z=0$ which is explicitly a contradiction when a pollution generating technology is supposed.

On the other hand, the DEA-MBP model introduced by Coelli, Lauwers [3], in spite of its advantages, has its own limitations when applied in different industries. The DEA-MBP model used by Coelli, Lauwers [3] for $N$ decision making units (DMU) is as below:

$$
\begin{aligned}
& \operatorname{Min}_{\lambda x_{o}^{e}} a x_{o}^{e} \\
& \text { s.t. } \\
& \sum_{n=1}^{N} \lambda_{n} x_{n i} \leq x_{o i}^{e} \quad i=1, \ldots, I \\
& \sum_{n=1}^{N} \lambda_{n} y_{n j} \geq y_{o j} \quad j=1, \ldots, J \\
& \lambda_{n} \geq 0, n=1, \ldots, N
\end{aligned}
$$

where the script $o$ denotes the under-assessment of DMU, and $x_{o}^{e}$ is the variable vector, which is being calculated to find the best composition of the inputs to generate the least pollution. $E$ is the nutrient vector.

There are several shortcomings in this DEA-MBP model. Firstly, this model neglects the real amount of the pollutants, which is hard to measure in the agricultural context. It is calculated by using the nutrient coefficient of inputs, such as the emission factor, whereas in many fields of 
study - for instance: electricity generation - the emission can be gauged directly. In addition, the model has a level of simplicity in using nutrients and cost coefficients to find the amount of the pollutants generated and the total cost of the production. This is a useful formulation for find trade-offs between the amount of the pollutants generated and the cost of the ingredients used. Nevertheless, this simple model cannot reflect the complexity existing in generation and disposing of the pollutants, like when abatement facilities are installed, or when a reward and charge mechanism, as mentioned in the calculation of total cost of fuel based on the fuel consumption rate ${ }^{4}$, is used. Furthermore, this model can be used only for the input orientation category of efficiency measurement. Finally, in case that the technology uses different ingredients to generate more than one pollutant, this model will still help find the optimal composition of the input required to generate the minimum amount of pollutant or run in the minimum cost condition. However, when the number of inputs is increased dramatically; the sensitivity of the model can be reduced and the validity of a DEA efficiency measurement system can be challenged seriously.

In sum, there remains a need for a more comprehensive eco-efficiency measurement model consistent with MBP. In addition, this type of models should not lose their comprehensiveness after MBP conditions are imposed. In the next section, an approach to incorporate MBP requirements in directional distance and slacks-based DEA models will be introduced.

\subsection{Incorporating MBP in DEA Models: a Discussion}

In this section, we present a full disscussion of the pros and cons of including MBP requirements into the slacks-based DEA and directional distance models. We focus on these types of models since this study aims to develop a more comprehensive and flexible MBP-enabled DEA model in order to measure ML index, and these two types of models are popular choices in measuring the ML index.

Slacks-Based measure of efficiecy was intially introduced by Färe and Grosskopf [11, 47] introduced a slacks-based model to measure the inefficiencies as below:

\footnotetext{
${ }^{4}$ In many industries, these types of incentives are imposed to control fuel which consumed and to force the industries to improve their combustion technologies or run them in their best condition.
} 


$$
\begin{aligned}
& D_{o}(x, y)=\operatorname{Max} \sum_{i=1}^{I} \alpha_{i}+\sum_{j=1}^{J} \beta_{j} \\
& \text { s.t. } \\
& \sum_{n=1}^{N} \lambda_{n} x_{i n} \leq x_{i o}-\alpha_{i} .1 ; i=1,2, \ldots, I \\
& \sum_{n=1}^{N} \lambda_{n} y_{j n} \geq y_{j o}+\beta_{j} .1 ; j=1,2, \ldots, J \\
& \lambda_{n} \geq 0 ; \beta_{j} \geq 0 ; \alpha_{i} \geq 0 ; n=1,2, \ldots, N ; i=1,2, \ldots, I ; j=1,2, \ldots, J
\end{aligned}
$$

We customize Model (3) so that it includes bad outputs as below ${ }^{5}$ :

$$
D_{o}(y, z)=\operatorname{Max} \sum_{j=1}^{J} \beta_{j}+\sum_{k=1}^{K} \gamma_{k}
$$

s.t.

$$
\begin{aligned}
& \sum_{n=1}^{N} \lambda_{n} x_{i n} \leq x_{i o} ; i=1,2, \ldots, I \\
& \sum_{n=1}^{N} \lambda_{n} y_{j n} \geq y_{j o}+\beta_{j} .1 ; j=1,2, \ldots, J \\
& \sum_{n=1}^{N} \lambda_{n} z_{k n}=z_{k o}-\gamma_{k} .1 ; k=1,2, \ldots, K \\
& \lambda_{n} \geq 0 ; \gamma_{k} \geq 0 ; \beta_{j} \geq 0 ; n=1,2, \ldots, N ; j=1,2, \ldots, J ; k=1,2, \ldots, K
\end{aligned}
$$

Here, $\alpha, \beta$ and $\gamma$ are the variables vectors, and $D_{o}$ denotes the distance of $\mathrm{DMU}_{o}$ from the frontier. A two-dimensional representation of Model (4) has been introduced by Ramli, Munisamy [48]. A complete economic and mathematical representation of Model (4) is addressed and applied in [46]. Here in Model (4), we use $(x, y+\beta, z-\gamma)$ to draw the DMU toward the eco-efficiency frontier. Thus, by replacing it in equation (1), we obtain: $z-\gamma=a^{\prime} x-b^{\prime}(y+\beta)$, which implies $z=a^{\prime} x-b^{\prime} y-b^{\prime} \beta+\gamma$. To be MBP- compatible, however, we should have $b^{\prime} \beta-\gamma=0$ or $b^{\prime} \beta=\gamma$. In this case, it means that the rate of decreasing the outputs should be equal to the rate of increasing the share of the pollutants in good outputs. For example if $b=0$, in the case of electricity generation, we get $\gamma=0$, since a firm cannot keep its inputs at the same level while increasing its good outputs and decreasing its bad outputs at the same time unless they adopt a more advanced technology ${ }^{6}$. This

\footnotetext{
${ }^{5}$ The third constraint guarantees null jointness property, which is defined as: if $(y, b) \in P(x)$ and $b=0$ then $y=0$. Good and bad outputs are produced jointly - see Chung, Färe, Grosskop (1990) [8]

${ }^{6}$ In here, the technology is assumed to be fixed.
} 
is possible only if the composition of inputs used is changed. Therefore, we customize Model (4) as below:

$$
\begin{aligned}
& D_{o}(x, y, z)=\operatorname{Max} \sum_{i=1}^{I} \alpha_{i}+\sum_{j=1}^{J} \beta_{j}+\sum_{k=1}^{K} \gamma_{k} \\
& \text { s.t. } \\
& \sum_{n=1}^{N} \lambda_{n} x_{i n} \leq x_{i o}-\alpha_{i} .1 ; i=1,2, \ldots, I \\
& \sum_{n=1}^{N} \lambda_{n} y_{j n} \geq y_{j o}+\beta_{j} .1 ; j=1,2, \ldots, J \\
& \sum_{n=1}^{N} \lambda_{n} z_{k n}=z_{k o}-\gamma_{k} .1 ; k=1,2, \ldots, K \\
& \lambda_{n} \geq 0 ; \alpha_{i} \geq 0 ; \beta_{j} \geq 0 ; \gamma_{k} \geq 0 ; n=1,2, \ldots, N ; \\
& i=1,2, \ldots, I ; j=1,2, \ldots, J ; k=1,2, \ldots, K
\end{aligned}
$$

Then, to be consistent with the MBP conditions, we should have $z-\gamma=a^{\prime}(x-\alpha)-b^{\prime}(y+\beta)$. This implies: $z=a^{\prime} x-b^{\prime} y-a^{\prime} \alpha-b^{\prime} \beta+\gamma$; therefore, it is compulsory to have $a^{\prime} \alpha=\gamma-b^{\prime} \beta$. In this case, if $b \neq 0$, then we get $\gamma-b^{\prime} \beta>0$ since $a^{\prime} \alpha>0$ for inefficient DMUs. This implies $\gamma>b^{\prime} \beta$, which means the decrease in pollutants should be strictly higher than the increase in the pollutant parts of the good outputs ( $b^{\prime} \beta$ is the amount of pollutant inside the good outputs). On the other hand, if $b=0$, then we obtain $a^{\prime} \alpha=\gamma$, which is the normal condition when good outputs do not generate any pollutants. As a result, Model (5) does not meet MBP requirements since the technology cannot expand the good and contract the bad with a drop in inputs. However, in the industries such as electricity, it is possible to do that with a change it the composition of inputs.

Now, considering the input-oriented model, we obtain:

$$
\begin{aligned}
& D_{o}(x, y, z)=\operatorname{Max} \sum_{i=1}^{I} \alpha_{i}+\sum_{k=1}^{K} \gamma_{k} \\
& \text { s.t. } \\
& \sum_{n=1}^{N} \lambda_{n} x_{i n} \leq x_{i o}-\alpha_{i} .1 ; i=1,2, \ldots, I \\
& \quad \sum_{n=1}^{N} \lambda_{n} y_{j n} \geq y_{j o} ; j=1,2, \ldots, J \\
& \quad \sum_{n=1}^{N} \lambda_{n} z_{k n}=z_{k o}-\gamma_{k} .1 ; k=1,2, \ldots, K \\
& \lambda_{n} \geq 0 ; \alpha_{i} \geq 0 ; \gamma_{k} \geq 0 ; n=1,2, \ldots, N ; i=1,2, \ldots, I ; j=1,2, \ldots, J ; k=1,2, \ldots, K
\end{aligned}
$$


Model (6) contracts inputs and bad outputs simultaneously. Again using equation (1), we achieve: $z-\gamma=a^{\prime}(x-\alpha)-b^{\prime} y$, which implies: $z=a^{\prime} x-b^{\prime} y-a^{\prime} \alpha+\gamma$. Therefore, to be MBP-compatible, it is necessary to have $-a^{\prime} \alpha+\gamma=0$. This implies $\gamma=a^{\prime} \alpha$, which is the ordinary condition if output remain constant since it guarantees that the rate of decrease in inputs and pollutants is identical.

We also introduce Model (7) as below:

$$
\begin{aligned}
& D_{o}^{\prime}(x, y, z)=\operatorname{Max} \theta \\
& \text { s.t. } \\
& \sum_{n=1}^{N} \lambda_{n} x_{i n} \leq x_{i o}-g_{x i} \cdot \theta ; i=1,2, \ldots, I \\
& \sum_{n=1}^{N} \lambda_{n} y_{j n} \geq y_{j o}+g_{y j} \cdot \theta ; j=1,2, \ldots, J \\
& \sum_{n=1}^{N} \lambda_{n} z_{k n}=z_{k o}-g_{b k} \cdot \theta ; k=1,2, \ldots, K \\
& \sum_{i=1}^{I} g_{x i}+\sum_{j=1}^{J} g_{y j}+\sum_{k=1}^{K} g_{b k}=1 \\
& \lambda_{n} \geq 0 ; g_{x i} \geq 0 ; g_{y k} \geq 0 ; g_{b j} \geq 0 ; n=1,2, \ldots, N ; \\
& i=1,2, \ldots, I, j=1,2, \ldots, J ; k=1,2, \ldots, K
\end{aligned}
$$

Model (7) is equivalent to Model (5). This can be easily verified by $g_{x i .} \theta=\alpha_{i}, g_{y j} . \theta=\beta_{j}$ and $g_{b k} . \theta$ $=\gamma_{k}$, where $g_{x i}, g_{y j}$ and $g_{b k}$ are the variable directions of the inputs, good and bad outputs, respectively.

On the other hand, in spite of the advantages of the aforementioned models, Coelli's DEA-MBP model has an advantage over all of them. The DEA-MBP model has been designed to identify the best composition of different fuel types so that the lowest amount of pollutants is generated. As it can be seen in the above discussion, there is a variety of models that meet the MBP requirements, and one can choose one or more of them based on the conditions. However, due to their nature, distance and slacks-based models fail to find the optimum composition of different fuel types to generate the lowest amount of pollutants since inputs are altered simultaneously.

We also adopt Model (8) from Briec [49], which incorporates bad outputs: 
s.t.

$$
\begin{aligned}
& \sum_{n=1}^{N} \lambda_{n} x_{i n} \leq x_{i o}\left(1-a_{i} . \theta\right) ; i=1,2, \ldots, I \\
& \sum_{n=1}^{N} \lambda_{n} y_{j n} \geq y_{j o}\left(1+b_{j} . \theta\right) ; j=1,2, \ldots, J \\
& \sum_{n=1}^{N} \lambda_{n} z_{k n}=z_{k o}\left(1-c_{k} . \theta\right) ; k=1,2, \ldots, K \\
& a_{i} \geq 0 ; b_{j} \geq 0 ; c_{k} \geq 0 ; \lambda_{n} \geq 0 ; \\
& n=1,2, \ldots, N ; i=1,2, \ldots, I ; j=1,2, \ldots, J ; k=1,2, \ldots, K
\end{aligned}
$$

where $a_{i}, b_{j}$ and $c_{k}$ contain the normalized input, good and bad outputs prices, which are called the orientation of the Farrell proportional distance. In our case, Materials Balance Principle, $a_{i}, b_{j}$ and $c_{k}$ are the same coefficients as in equation (1) with $c_{k}=1$ for $k=1, \ldots, K$. These coefficients, instead of the price information of Briec's model, reflect the pollutant parts of inputs and output. Similar to the prices, it is of our interest to keep the pollutants at the minimum level. Without loss of generality, here we assume constant return to scale contrary to Briec's original model. By $x_{i o} . a_{i}=g_{x i}, y_{j o} . b_{j}=g_{y j}$ and $z_{k o}=g_{z k}$, we can see that Model (7) and (8) are equivalents ${ }^{7}$. Accordingly, one can observe that the directions in Model (7) can reflect the magnitude of the pollutant part of the inputs and outputs, but in their normalized form; see equation (6) in Briec [49].

The discussion opened up in this section sheds light on incorporation of MBP in directional distance and slacks-based models. In the next section, a more comprehensive model is going to be introduced to incorporate MBP in DEA models for eco-efficiency measurement.

\subsection{An Alternative DEA-MBP Model for Eco-Efficiency Measurement}

To formulate eco-efficiency measurement problems incorporating MBP, we categorize inputs into high pollutants and low pollutant ones and introduce the following model:

\footnotetext{
${ }^{7}$ Here, we can omit $\sum_{i=1}^{I} g_{x i}+\sum_{j=1}^{J} g_{y j}+\sum_{k=1}^{K} g_{b k}=1$, which does not change the frontier, but plays the role of a scaling constraint to keep inefficiency variable, $\theta$, within the limit of $[0,1]$.
} 


$$
\begin{aligned}
& D_{o}(x, y, z)=\operatorname{Max} \sum_{l=1}^{L} \alpha l_{l}+\sum_{h=1}^{H} \alpha h_{h}+\sum_{m=1}^{M} \alpha_{m}+\sum_{j=1}^{J} \beta_{j}+\sum_{k=1}^{K} \gamma_{k} \\
& \text { s.t. } \\
& \sum_{n=1}^{N} \lambda_{n} x l_{l n} \leq x l_{l o}+\alpha l_{l} .1 ; l=1,2, \ldots, L \\
& \sum_{n=1}^{N} \lambda_{n} x h_{h n} \leq x h_{h o}-\alpha h_{h} .1 ; h=1,2, \ldots, H \\
& \sum_{n=1}^{N} \lambda_{n} x_{n} \leq x_{m o}-\alpha_{m} .1 ; m=1,2, \ldots, M \\
& \sum_{n=1}^{N} \lambda_{n} y_{j n} \geq y_{j o}+\beta_{j} .1 ; j=1,2, \ldots, J \\
& \sum_{n=1}^{N} \lambda_{n} z_{k n}=z_{k o}-\gamma_{k} .1 ; k=1,2, \ldots, K \\
& \sum_{l=1}^{L} \alpha l_{l}-\sum_{h=1}^{H} \alpha h_{h}=0 \\
& \gamma_{k}-\sum_{j=1}^{J} b_{j k} \beta_{j}=\sum_{h=1}^{H} a h_{h k} \alpha h_{h}-\sum_{l=1}^{L} a l_{l k} \alpha l_{l} ; k=1,2, \ldots, K \\
& \lambda_{n} \geq 0 ; \alpha l_{l} \geq 0 ; \alpha h_{h} \geq 0 ; \alpha_{m} \geq 0 ; \beta_{j} \geq 0 ; \gamma_{k} \geq 0 ; n=1,2, \ldots, N ; \\
& l=1,2, \ldots, L ; h=1,2, \ldots, H ; m=1,2, \ldots, M ; j=1,2, \ldots, J ; k=1,2, \ldots, K
\end{aligned}
$$

where, $x h$ and $x l$ denote high pollutants and low pollutant inputs determined by the magnitude of their pollutant part. The parameter $x$ represents the nonpolluting inputs such as capital. As such, $\alpha h$ and $\alpha l$ are defined as the rate of contraction and expansion of high and low pollutant inputs, respectively; and $\alpha$ is the rate of contraction in nonpolluting inputs. Also, $a h$ and $a l$ are the pollutant part of the high and low pollutant inputs, respectively. It is evident that $a h>a l$; and if $a h=a l$, there will be no need for distinction between high and low pollutants. Consequently, we should have $H+L+M=I$, the total number of inputs. As a requirement for every mathematical programming model, it can be simply proven that Model (9) is feasible. Toward this aim, (9-1) to (9-5) are conventional slacks-based model adopted from Färe and Grosskopf [11], Färe and Grosskopf [47]. The Constraint (9-6) is also consistent, since at least for the efficient DMUs, we have $\alpha h=\alpha l=0$ for all $h=1,2, \ldots, H$ and $l=1,2, \ldots, L$, which implies $\sum_{l=1}^{L} \alpha l_{l}-\sum_{h=1}^{H} \alpha h_{h}=0$. Besides, the Constraint (9-7) is also consistent with other constraints; otherwise, there would be no DMU in PPS, which can operate under the first law of thermodynamics.

In Model (9), (9-1) and (9-2) represent a tendency for the model to increase the consumption of the low pollutant input and decrease the high pollutant one simultaneously. This is accompanied by (9-6), which guarantees that at most the actual amount of inputs is consumed to generate at least the same amount of good outputs and at most the same amount of pollutants. This is while 
it tries to increase low pollutant inputs and decrease the high pollutant ones simultaneously (This property is achieved by the first and second constraints). (9-3) and (9-5) are the conventional constraints of the adopted slacks-based model, Model (5), imposed on the nonpolluting inputs, good outputs, and the bad ones, respectively. Finally, (9-7) verifies compatibility of the MBP (It can be seen that (9-7) will be neutral if $k$ is a non-polluting bad output). An in-depth discussion on incorporation of MBP constraint is ensued below.

The vector $(x h-\alpha h, x l+\alpha l, y+\beta, z-\gamma)$ should be in the PPS. Thus, testing with equation (1), we obtain: $z=a h^{\prime} \cdot x h+a l^{\prime} \cdot x l-b^{\prime} \cdot y+\gamma-a h^{\prime} . \alpha h+a l^{\prime} . \alpha h-b^{\prime} \beta$; then, to be MBP-consistent, we should have:

$$
\gamma-b^{\prime} \beta=a h^{\prime} . \alpha h-a l^{\prime} . \alpha l
$$

where $a h^{\prime}>a l^{\prime}$, together with (9-6), implies that the right hand side of (10) is strictly positive. Thus, the drop in the total amount of the pollutants - the left hand side - should be equal to drop in the pollutant part of the inputs. This is because, $b^{\prime} \beta$ as the pollutant part of the good output, remains constant since the amount of the inputs has been kept constant by (9-6), implying $\gamma>b^{\prime} \beta$. As a result, drop in pollution should be strictly higher than the growth in the pollution part of the good output, which is of favor. It is worthwhile to say that Model (7) and Model (8) can also be customized as Model (9). The distance function for these two models falls within the unity interval, $[0,1]$.

Toward operationalization, we adopt Model (9) to see the trend of Iranian gas power plant eco-

efficiency changes during an eight-year period, 2003-2008, using the ML index. Details are discussed in the next section.

\section{Results}

\subsection{An Application in Power Plant Eco-efficiency Measurement}

We use data of Iranian gas-fired power plants from 2003 to 2010 to measure ML index, employing Model (9). General formulation of ML index is adopted from [8] and we also employed the approach introduced in Arabi, Munisamy [50] to overcome the prevalent infeasibility issue occurs in MLI measurement. The eco-efficiency measurement factors are summarized in Table 1 below: 
Table 1: Definitions of Input-Output Factors

\begin{tabular}{|c|c|}
\hline Inputs & Definition \\
\hline 1. Operational Availability & $\begin{array}{l}\text { Yearly average of a power plant capacity declared to the National Dispatching Unit available during the daily peak } \\
\text { hours.* }\end{array}$ \\
\hline $\begin{array}{l}\text { Fuel: } \\
\text { 2.Gas } \\
\text { 3.Gasoil }\end{array}$ & $\begin{array}{l}\text { Total Gas, Gasoil and Fuel Oil consumed by a power plant in a year*. Each is calculated by: } \\
\text { Gas (Calorie)=Gas Heating Value (Calorie/M3)* Total Gas Combusted by a power plant in a year (M3) } \\
\text { Gasoil (Calorie)=Gasoil Heating Value (Calorie/Liter)* Total Gasoil Combusted by a power plant in a year (Liter) }\end{array}$ \\
\hline Outputs & Definition \\
\hline $\begin{array}{l}\text { Undesirable: } \\
\text { 1.SO2 }\end{array}$ & Total tons of SO2 generated by a power plant in a year ** \\
\hline $\begin{array}{l}\text { 2. Deviation from } \\
\text { Generation plan }\end{array}$ & $\begin{array}{l}\text { Summation of daily ratios of generated energy, to the energy supposed to be measured by national dispatching } \\
\text { during peak hour in a year. } * * *\end{array}$ \\
\hline $\begin{array}{l}\text { Desirable: } \\
\text { Generated Energy }\end{array}$ & Mega Watt Hours of energy generated by a power plant and injected to the National Power Grid in a year* \\
\hline
\end{tabular}

In this study, $\mathrm{SO} 2$ has been considered a proxy for all gases emitted. Many similar researches such as Golany, Roll [51], Korhonen and Luptacik [52], Färe, Grosskopf [53], and Burnett and Hansen [54] have incorporated SO2 as an emission factor to measure the eco-efficiency of power plants. This gas is a major cause of acid rains and has a predominant role in human respiratory diseases. The data on SO2 emission have been acquired from Tavanir Environmental Affairs Bureau. Therefore, emission is signified by the yearly SO2 produced by each power plant in tons. According to the country's energy balance sheet in annual reports, power generation sector has produced 192733 tons of SO2 in 2005 and this amount has increased to 497354 in 2009. This is while the contribution of power plants to $\mathrm{SO} 2$ production amongst all energy industries has been increased from $23.01 \%$ to $36.68 \%$ during the same years.

It is worth noting that in the electricity generation case, $b=0$ because electricity as the sole good output does not contain any emission factors such as Sulfur, which can lead to production of additional Soz. In addition, to check the robustness of Model (9), we also fulfill material flow requirement as a trade-off between inputs and outputs. This type of trade-offs has already been introduced to DEA literature by Podinovski [55]. In our case, there is a trade-off between polluting inputs and good and bad outputs. Based on the Podinovski's formulations, MBP type trade-offs can be written as below:

$$
\left(P H_{t}, P L_{t}, Q_{t}, R_{t}\right), \quad t=1,2, \ldots, T \text {. }
$$

where $P H_{t}, P L_{t}, Q_{t}$, and $R_{t}$ are corresponding trade-off vectors to high polluting inputs, low polluting inputs (including nonpolluting), good and bad outputs, respectively. Moreover, $t$ 
denotes the number of trade-off relationship. In our case, we have three inputs; namely, operational availability, gas, and gasoil, the last two of which are polluting, and gasoil is even more polluting than gas. There are two outputs, here: the generated energy, which is a single nonpolluting good output, and $\mathrm{So}_{2}$, as a single bad polluting output. Thus, we can formulate three trade-offs for each polluting input. Following the notation of Podinovski [55], we can formulate the trade-offs between gas, gasoil, fuel oil and SO2 as below:

$$
P L_{l}=(1), P H_{1}=(0,-1)^{T}, Q_{1}=(0), R_{l}=\left(a_{1}-a_{2}, 0\right)^{T}
$$

where $a_{1}, a_{2}$ are gas and gasoil emission factors in Iran, respectively ${ }^{8}$. By (12), one can discern from (12) that it would be a cleaner strategy if with one more calorie of gas, one less calorie of gasoil were combusted. Thus, generation of the same level of the electricity would result in lower amount of emissions. That is to say, emission of SO2 would drop by $a_{1}-a_{2}$ in tons.

Now, by adding Podinovski’s trade-off formulations to Model (5), we obtain:

$$
\begin{aligned}
& \text { TO-Do }(x, y, z)=\operatorname{Max} \sum_{i=1}^{I} \alpha l_{l}+\sum_{i=1}^{I} \alpha h_{h}+\sum_{j=1}^{J} \beta_{j}+\sum_{k=1}^{K} \gamma_{k} \\
& \text { s.t. } \\
& \sum_{n=1}^{N} \lambda_{n} x l_{l n}+\sum_{t=1}^{T} \pi_{t} P L_{l t} \leq x l_{l o}-\alpha l_{l} .1 ; l=1,2, \ldots, L \\
& \sum_{n=1}^{N} \lambda_{n} x h_{h n}+\sum_{t=1}^{T} \pi_{t} P H_{h t} \leq x h_{h o}-\alpha h_{h} .1 ; h=1,2, \ldots, H \\
& \sum_{n=1}^{N} \lambda_{n} y_{j n}+\sum_{t=1}^{T} \pi_{t} Q_{j t} \geq y_{j o}+\beta_{j} .1 ; j=1,2, \ldots, J \\
& \sum_{n=1}^{N} \lambda_{n} z_{k n}+\sum_{t=1}^{T} \pi_{t} R_{k t}=z_{k o}-\gamma_{k} .1 ; k=1,2, \ldots, K \\
& \lambda_{n} \geq 0 ; \alpha l_{l} \geq 0 ; \alpha h_{h} \geq 0 ; \beta_{j} \geq 0 ; \gamma_{k} \geq 0 ; \pi_{t} \geq 0 ; n=1,2, \ldots, N ; \\
& l=1,2, \ldots, L ; h=1,2, \ldots, H ; j=1,2, \ldots, J ; k=1,2, \ldots, K ; t=1,2, \ldots, T
\end{aligned}
$$

$8 a_{1}=-1.86610 \mathrm{E}-06$ Tone/Cal and $a_{2}=-6.84500 \mathrm{E}-06$ Tone/Cal. See Result of the Comprehensive Plan of Tehran Air Pollution Control, 1997, by JICA and Municipality of Tehran. 
where $\pi_{t}$ is corresponding trade-off variable and $T O-D_{o}$ stands for trade-off distance of the unit under assessment, $\mathrm{DMU}_{o}$. Using an identical proof, it can be easily verified that PPS of Model (13) satisfies axioms A1 to A5 in Podinovski [55]. Thus Model (13) is suitable for operationalization; thereby, it can be used to verify the robustness of Model (9) as it possesses MBP characteristics.

\section{Discussions}

We ran Model (9) and Model (13), using AIMMS 3.12 to calculate efficiency scores and ML indices. The Results are given in Table 2 below:

Table 2: Efficiency scores of Iranian Gas Power Plants in an 8-year Period

\begin{tabular}{|c|c|c|c|c|c|c|c|c|c|c|c|c|c|c|c|c|}
\hline \multirow{2}{*}{$\begin{array}{c}\text { Gas Power } \\
\text { Plants } \\
\text { Codes }\end{array}$} & \multicolumn{8}{|c|}{ Efficiency Scores, results of Model (9) } & \multicolumn{8}{|c|}{ Efficiency, results of Model (13) } \\
\hline & 2003 & 2004 & 2005 & 2006 & 2007 & 2008 & 2009 & 2010 & 2003 & 2004 & 2005 & 2006 & 2007 & 2008 & 2009 & 2010 \\
\hline H_1 & 1.0000 & & & & & & & & 0000 & & & & & & & \\
\hline H_2 & & 1.0000 & 0.9403 & 1.0000 & 0.9561 & & & & & & & 1.0000 & 0.9694 & 0.9789 & 1.0000 & 0.938 \\
\hline $\mathrm{H} \_3$ & & & 0.8905 & & 0.9037 & & & & & & & & & 1.0000 & & \\
\hline H_4 & & 1.0000 & 1.0000 & .0000 & 1.0000 & 1.0000 & & & 0000 & & 1.0000 & & 0.8978 & 1.0000 & 0.9511 & 1.0000 \\
\hline H_5 & 9645 & 0.9407 & 0.9345 & 9372 & 0.9204 & 9126 & 0.9665 & & & 0.9708 & 0.9771 & 0.9 & 3624 & 1.0000 & 592 & 0.879 \\
\hline H_6 & & & 0.9764 & & 1.0000 & & & & & & & & & & & \\
\hline H_7 & 0000 & .0000 & 1.0000 & & 1.0000 & .0000 & & & 0000 & & 1.0000 & & & 0.9019 & & 1.0 \\
\hline H_8 & 0.8864 & 0.8238 & 0.8332 & 1.0000 & 1.0000 & 1.0000 & 0.8475 & 0.8213 & .8438 & 1.0000 & 1.0000 & 1.0000 & .0000 & 1.0000 & 1.0000 & 1.0000 \\
\hline & & & & & & & & & 1.0000 & & & & & & & \\
\hline H_10 & 8971 & 584 & 0.8603 & 0000 & 0.7837 & 0.9037 & 1.0000 & 0.9522 & 0.8515 & 1.0000 & 1.0000 & 1.0000 & 1.0000 & 1.0000 & 1.0000 & 0.875 \\
\hline H_11 & 9299 & 0.9484 & 0.9387 & 0.9326 & 0.9246 & 0.9204 & 0.9394 & & 0.9054 & 0.8910 & 1.0000 & 0.6755 & 0.8698 & 1.0000 & 0.8630 & 0.776 \\
\hline $\mathrm{H} \_12$ & & & & & & & & & & & & & & & & \\
\hline & 8232 & 0.7710 & 0.7308 & 0.7399 & 0.6680 & 6985 & 0.7065 & & 0.6954 & 0.9169 & 0.7436 & & & 0.9888 & 1.0000 & 0.826 \\
\hline H_14 & 8927 & 0.9127 & 0.8850 & 0.8838 & 0.9030 & 0.9085 & 0.8594 & 0.8492 & 0.8273 & 0.8179 & 0.8249 & 0.8793 & 1.0000 & 1.0000 & 0.7451 & 0.7252 \\
\hline H_15 & 0.9516 & 0.9488 & 0.9426 & 0.9375 & 1.0000 & 1.0000 & 0.9405 & 0.9099 & 0.8851 & 0.9236 & 0.8178 & 1.0000 & 1.0000 & 1.0000 & 1.0000 & 0.9054 \\
\hline H_16 & 000 & 0.9532 & 0.9207 & 0.9715 & 1.0000 & 0000 & 1.0000 & 0.9 & 1.0000 & 0.8380 & 1.0000 & 1.0000 & 1.0000 & 1.0000 & 1.0000 & 0.9500 \\
\hline H_17 & 1.0000 & 1.0000 & 1.0000 & 1.0000 & 1.0000 & 0.9012 & 0.7036 & & 1.0000 & 1.0000 & 1.0000 & 1.0000 & 1.0000 & 0.8454 & 1.0000 & 0.9104 \\
\hline orrelation & 0.9769 & 8893 & 0.9185 & 0.8584 & 0.9198 & 0.9148 & 0.7049 & & & & & & & & & \\
\hline
\end{tabular}

As it can be seen in Table 2, efficiency scores calculated using Model (9) and Model (13) slacksbased measures are highly correlated. Trade-Off (TO) model (Model 13) shows a relatively lower efficiency score than the one MBP model (Model 9) does. The reason is that the production possibility set with trade-offs is larger than the one without trade-offs. This is itself a result of introducing new trade-off variables to the model, here $P L, P H$ and $R$ (See Podinovski [55]). In addition, Model (9) imposes two extra constraints, (9-6), fuel control and (9-7), MBP 
condition, which tighten the feasible region. Consequently, efficiency scores derived from Model (9) should be higher than the same scores when derived from Model (13) in most of the cases. In our case, 87 out of 136 efficiency scores, which are displayed in bold in Table 2, calculated by slacks-based measure from MBP model are higher than, or equal to the same score derived using TO model.

Figure 1 below depicts a similar trend in both models. The correlation between these two series is high at 0.817 .

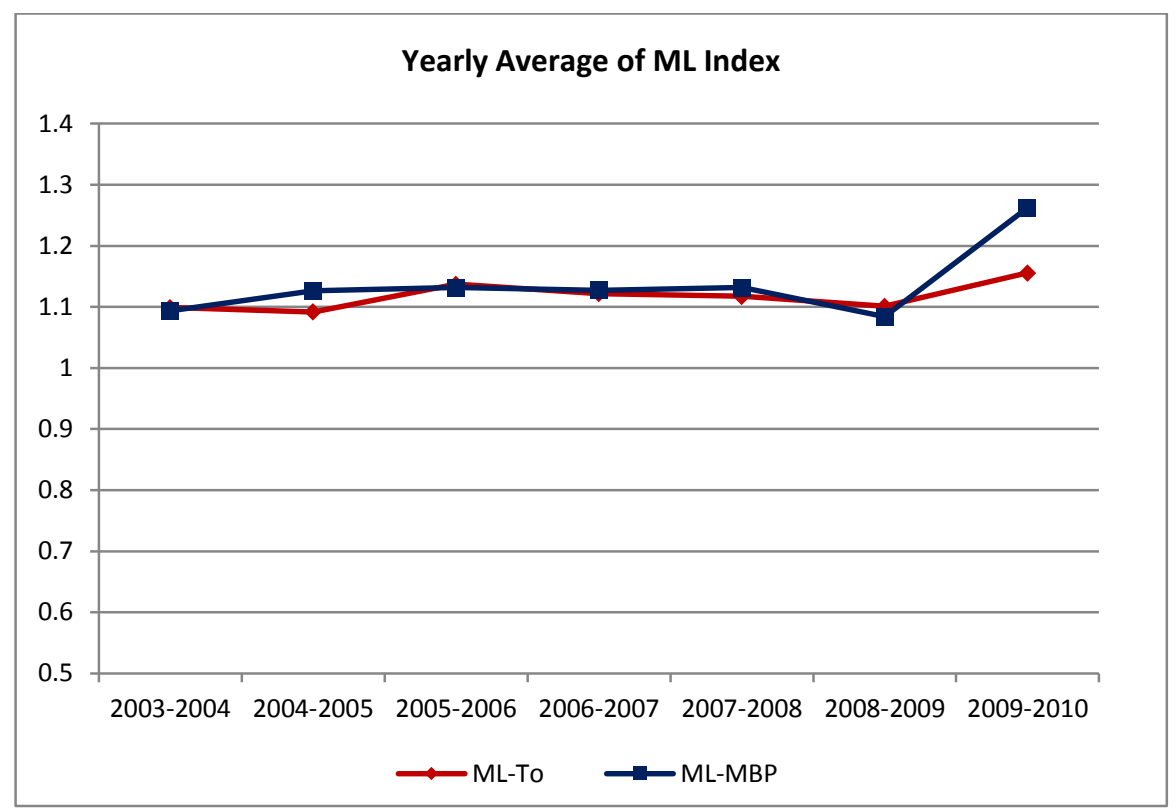

Figure 1: Yearly Average of ML Index

To see the eco-efficiency trend for all gas power plants, we also use ML index to calculate the aggregated rate of change for each period, $S_{M L}$, using effective capacity of the power plants as follows:

$$
S_{M L}=\sum_{n=1}^{N}\left(M L_{n} \cdot P E F F C A P_{n}-\operatorname{PEFFCAP}_{n}\right) / \sum_{n=1}^{N} \operatorname{PEFFCAP}_{n}
$$

where:

$M L_{n}=\mathrm{ML}$ index rate for $\mathrm{n}^{\text {th }}$ power plant in a particular period, ML is a contemporaneous index PEFFCAP ${ }_{n}=$ Effective Capacity for $\mathrm{n}^{\text {th }}$ power plant in a particular period $S_{M L}=$ Aggregated Rate of Change of ML index by Effective Capacity 
The results are depicted in the following graph:

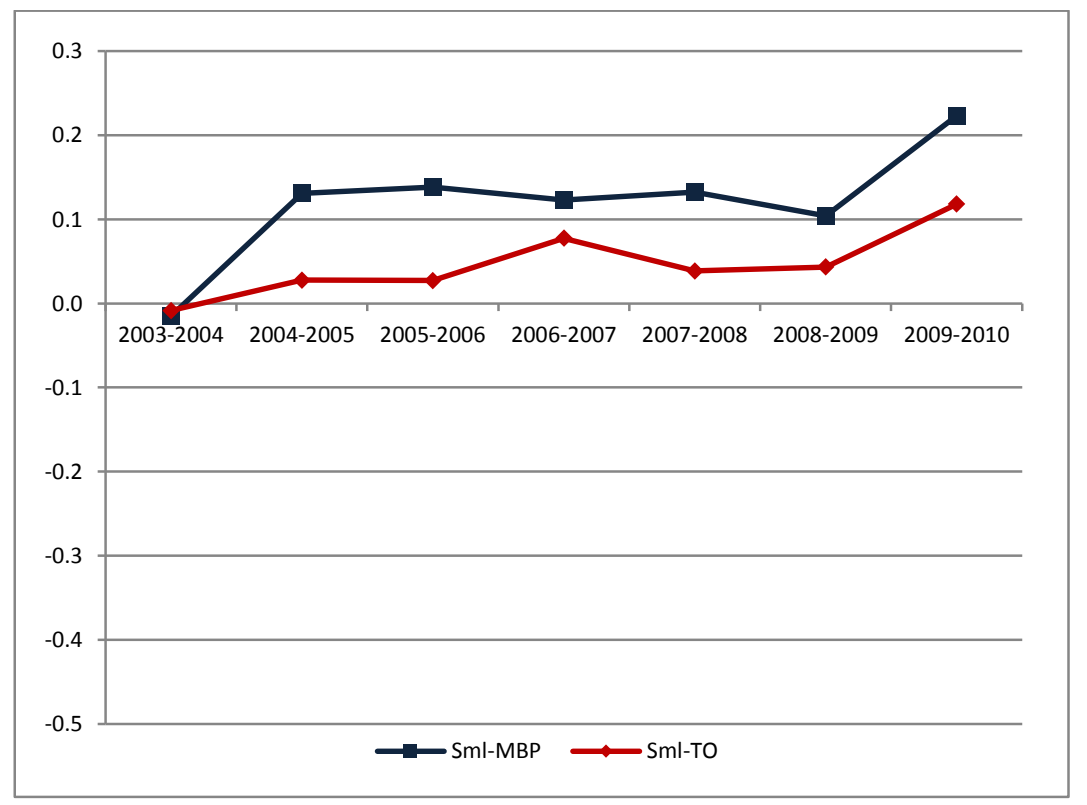

Figure 2: $\mathbf{S}_{\mathbf{M L}}$ for Iranian Gas Power Plants over an Eight-Year Period

In addition to the similarity between the results of the two models, Figure 2 exhibits a clear enhancement in gas power plant eco-efficiency during the period. Although more fluctuation is observed in comparison with Figure 1, correlation between the two series is very high at 0.8951 , even higher than that of the series in Figure 1.

In this paper, we addressed the difficulties of incorporating the Material Balance Principle in the directional distance and slacks-based models. Due to the complexity existing heretofore, there are grey areas in real systems that have remained unexplored in the non-parametric frontier models. With the increasing environmental concerns, the accordance of performance measurement systems with the nature of the system under evaluation has become a necessity. MBP is one of the first and vital conditions to check and decide whether or not the eco-efficiency measurement model is compatible with the nature of the system.

Due to the complexity of real systems as discussed in Section 2.1, the majority of the previous eco-efficiency measurement methods either neglected MBP or failed to incorporate it successfully. Thus, different MPB aspects and circumstances have remained unknown or unexplored. One of the earliest DEA approaches, which is consistent with MBP, was introduced by Coelli, Lauwers [3]. In Section 2.3, we addressed the requirements for a more feasible eco- 
efficiency model incorporating MPB by verifying the strengths and weaknesses of the model introduced by Coelli, Lauwers [3]. In so doing, using a simple equation given by Coelli, Lauwers [3], we verified the MPB compatibility of one of the most popular DDF models introduced by Chung, Färe [8] for measurement of ML index.

In section 2.3, consistency of a number of adopted DDF and slacks-based models with MBP was checked and presented using Coelli's equation and the conditions for MBP capability for all of these models. We presented three similar models: Models (5), (7) and (8), which can be used interchangeably. However, it became evident that the DDF and slacks-based models are not capable of finding the target DMU based on the best composition of the polluting inputs combusted. Therefore, these series of model may fail to evaluate inefficient DMUs unlike Coelli's DEA-MBP model.

In Section 2.5, we introduced Model (9) with MBP capability through adopting Model (7). In addition to its MBP capability, Model (9) became capable of letting low polluting input to increase and simultaneously forcing high polluting ones to decrease once the inputs were divided into high and low polluting inputs. This rotation in polluting inputs is controlled by constraint (96) to guarantee that the same volume of ingredients needed is delivered to the system so that the same good outputs are generated as before. However, if other constraints allowed, the generated pollution would be less than it used to be. This property of the model helps find the target DMU based on the best polluting inputs composition. Besides, it has MBP capability and can project the DMU to a point on the frontier with more good outputs and less bad outputs generated. These all culminate in construction of a model, which possesses more comprehensive properties. This is while the previous models have not had all of these properties all at once.

Finally, Model (9) was compared to another similar model adopted from Model (7) by adding the MPB requirements as trade-offs between polluting inputs and polluting bad outputs. Both models were used to measure ML index of Iranian gas power plants during an eight-year period, 20032010. The results showed a similar trend for both models. This similarity confirms the robustness of Model (13). 


\section{Conclusion and Policy Implications}

In this paper, we investigated the conditions for incorporating Material Balance Principle in some conventional eco-efficiency DEA models. Many of these models start to suffer from deficiencies when MBP conditions are included. We also addressed some drawbacks of Coelli's DEA-MBP model and introduced a more flexible and comprehensive model; that is, a MBPcompatible model called the MBP Malmquist Luenberger Index. This model was successfully applied to measure ML index for Iranian gas power plants over a span of eight years. The results obtained using this model were compared with those obtained using another slacks-based DEA model incorporating MBP with trade-offs. The comparison showed a high correlation between the two, thus confirming the robustness and applicability of our model. Researchers interested could adopt the proposed approach to deal with other DEA models.

\section{References}

[1] Lauwers L. Justifying the incorporation of the materials balance principle into frontier-based eco-efficiency models. Ecological Economics. 2009;68(6):1605-14.

[2] Baumgärtner S, Dyckhoff H, Faber M, Proops J, Schiller J. The concept of joint production and ecological economics. Ecological Economics. 2001;36(3):365-72.

[3] Coelli T, Lauwers L, Huylenbroeck G. Environmental efficiency measurement and the materials balance condition. Journal of Productivity Analysis. 2007;28(1-2):3-12.

[4] Tyteca D. Linear Programming Models for the Measurement of Environmental Performance of Firms - Concepts and Empirical Results. Journal of Productivity Analysis. 1997;8(2):183-97.

[5] Fare R, Grosskopf S, Lovell CAK, Pasurka C. Multilateral Productivity Comparisons When Some Outputs are Undesirable: A Nonparametric Approach. The Review of Economics and Statistics. 1989;71(1):90-8.

[6] Färe R, Grosskopf S, Tyteca D. An activity analysis model of the environmental performance of firms - application to fossil-fuel-fired electric utilities. Ecological Economics. 1996;18(2):161-75.

[7] Reinhard S, Knox Lovell CA, Thijssen GJ. Environmental efficiency with multiple environmentally detrimental variables; estimated with SFA and DEA. European Journal of Operational Research. 2000;121(2):287-303.

[8] Chung YH, Färe R, Grosskopf S. Productivity and Undesirable Outputs: A Directional Distance Function Approach. Journal of Environmental Management. 1997;51(3):229-40.

[9] Shephard RW, Gale D, Kuhn HW. Theory of cost and production functions: Princeton University Press Princeton, NJ, 1970.

[10] Färe R, Grosskopf S, Pasurka JCA. Environmental production functions and environmental directional distance functions. Energy. 2007;32(7):1055-66.

[11] Färe R, Grosskopf S. Directional distance functions and slacks-based measures of efficiency. European journal of operational research. 2010a;200(1):320-2.

[12] Picazo-Tadeo AJ, Reig-Martínez E, Hernández-Sancho F. Directional distance functions and environmental regulation. Resource and Energy Economics. 2005;27(2):131-42. 
[13] Diabat A, Shetty U, Pakkala T. Improved efficiency measures through directional distance formulation of data envelopment analysis. Annals of Operations Research. 2013:1-22.

[14] Yang C-C. An enhanced DEA model for decomposition of technical efficiency in banking. Annals of Operations Research. 2014;214(1):167-85.

[15] Ayres RU, Kneese AV. Production, Consumption, and Externalities. The American Economic Review. 1969;59(3):282-97.

[16] Van der Hamsvoort CPCM, Latacz-Lohmann U. Sustainability: a review of the debate and an extension. International Journal of Sustainable Development and World Ecology. 1998;5:99-110.

[17] Field BC. "Environmental Economics. An Introduction ". Economics Series, Mc Graw Hill. 1994.

[18] Field B, Olewiler N. Environmental Economics (Updated second Canadian edition). McGraw-Hill Ryerson Limited. Canada; 2005.

[19] Tyteca D. Linear programming models for the measurement of environmental performance of firms — concepts and empirical results. Journal of Productivity Analysis. 1997a;8(2):183-97.

[20] Lauwers L, Van Huylenbroeck G, Rogiers G. Technical, economic and environmental efficiency analysis of pig fattening farms, Poster presentation at the 9th European Congress of Agricultural Economists, Warschau. Polen, August 24th-28th. 1999.

[21] Murty S, Robert Russell R, Levkoff SB. On modeling pollution-generating technologies. Journal of Environmental Economics and Management. 2012.

[22] Pethig R. Non-linear production, abatement, pollution and materials balance reconsidered. Journal of Environmental Economics and Management. 2006;51(2):185-204.

[23] Färe R, Grosskopf S, Pasurka Jr C. Joint Production of Good and Bad Outputs with a Network Application. Encyclopedia of Energy, Natural Resources and Environmental Economics. 2011.

[24] Kuosmanen N, Kuosmanen T. Modeling Cumulative Effects of Nutrient Surpluses in Agriculture: A Dynamic Approach to Material Balance Accounting. Ecological Economics. 2013;90:159-67.

[25] Utlu Z, Kincay O. An assessment of a pulp and paper mill through energy and exergy analyses. Energy. 2013;57:565-73.

[26] Thanh Nguyen T, Hoang VN, Seo B. Cost and environmental efficiency of rice farms in South Korea. Agricultural Economics. 2012;43(4):369-78.

[27] Farrell MJ. The measurement of productive efficiency. Journal of the Royal Statistical Society Series A (General). 1957;120(3):253-90.

[28] Charnes A, Cooper W, Rhodes E. Measuring the efficiency of decision making units. European journal of operational research. 1978;2(6):429-44.

[29] Banker RD, Charnes A, Cooper WW. Some models for estimating technical and scale inefficiencies in data envelopment analysis. Management Science. 1984;30(9):1078-92.

[30] Zhou G, Chung W, Zhang X. A study of carbon dioxide emissions performance of China's transport sector. Energy. 2013.

[31] Khoshroo A, Mulwa R, Emrouznejad A, Arabi B. A non-parametric Data Envelopment Analysis approach for improving energy efficiency of grape production. Energy. 2013;63:189-94.

[32] Arabi B, Munisamy S, Emrouznejad A, Shadman F. Power industry restructuring and eco-efficiency changes: A new slacks-based model in Malmquist-Luenberger Index measurement. Energy Policy. 2014;68:132-45.

[33] Wei Y-M, Liao H, Fan Y. An empirical analysis of energy efficiency in China's iron and steel sector. Energy. 2007;32(12):2262-70.

[34] Emrouznejad A, Parker BR, Tavares G. Evaluation of research in efficiency and productivity: A survey and analysis of the first 30 years of scholarly literature in DEA. Socio-Economic Planning Sciences. 2008;42(3):151-7. 
[35] Seiford LM. Data envelopment analysis: The evolution of the state of the art (1978-1995). Journal of Productivity Analysis. 1996;7(2):99-137.

[36] Emrouznejad A. Advances in data envelopment analysis. Annals of operations research. 2014;214(1):1-4.

[37] Berg SA, Førsund FR, Jansen ES. Malmquist indices of productivity growth during the deregulation of Norwegian banking, 1980-89. The Scandinavian Journal of Economics. 1992;94:211-28.

[38] Hadi Vencheh A, Kazemi Matin R, Tavassoli Kajani M. Undesirable factors in efficiency measurement. Applied Mathematics and Computation. 2005;163(2):547-52.

[39] Huang X, Hu D, Zhou Z. Measuring efficiency in Chinese commercial banks using a DEA model with undesirable output. International Journal of Information and Decision Sciences. 2013;5(2):140-53.

[40] Jahanshahloo GR, Lotfi FH, Shoja N, Tohidi G, Razavyan S. Undesirable inputs and outputs in DEA models. Applied Mathematics and Computation. 2005;169(2):917-25.

[41] Tone K. A slacks-based measure of efficiency in data envelopment analysis. European journal of operational research. 2001;130(3):498-509.

[42] Zhou P, Poh KL, Ang BW. A non-radial DEA approach to measuring environmental performance. European journal of operational research. 2007;178(1):1-9.

[43] Lozano S, Gutiérrez E, Moreno P. Network DEA approach to airports performance assessment considering undesirable outputs. Applied Mathematical Modelling. 2013;37(4):1665-76.

[44] Bilsel M, Davutyan N. Hospital efficiency with risk adjusted mortality as undesirable output: the Turkish case. Annals of Operations Research. 2014;221(1):73-88.

[45] Munisamy S, Arabi B. Eco-efficiency Change in Power Plants: Using A Slacks-Based Measure for the MetaFrontier Malmquist Luenberger Productivity Index. Journal of Cleaner Production. 2015.

[46] Welch E, Barnum D. Joint environmental and cost efficiency analysis of electricity generation. Ecological Economics. 2009;68(8-9):2336-43.

[47] Färe R, Grosskopf S. Directional distance functions and slacks-based measures of efficiency: Some clarifications. European journal of operational research. 2010b;206(3):702.

[48] Ramli NA, Munisamy S, Arabi B. Scale directional distance function and its application to the measurement of ecoefficiency in the manufacturing sector. Annals of Operations Research. 2013:1-18.

[49] Briec W. A graph-type extension of Farrell technical efficiency measure. Journal of Productivity Analysis. 1997;8(1):95-110.

[50] Arabi B, Munisamy S, Emrouznejad A. A new slacks-based measure of Malmquist-Luenberger index in the presence of undesirable outputs. Omega. 2015;51(0):29-37.

[51] Golany B, Roll Y, Rybak D. Measuring efficiency of power plants in Israel by data envelopment analysis. Engineering Management, IEEE Transactions on. 1994;41(3):291-301.

[52] Korhonen PJ, Luptacik M. Eco-efficiency analysis of power plants: an extension of data envelopment analysis. European Journal of Operational Research. 2004;154(2):437-46.

[53] Färe R, Grosskopf S, Noh D-W, Weber W. Characteristics of a polluting technology: theory and practice. journal of Econometrics. 2005;126(2):469-92.

[54] Burnett RD, Hansen DR. Ecoefficiency: Defining a role for environmental cost management. Accounting, Organizations and Society. 2008;33(6):551-81.

[55] Podinovski V. Production trade-offs and weight restrictions in data envelopment analysis. Journal of the operational Research Society. 2004;55(12):1311-22. 